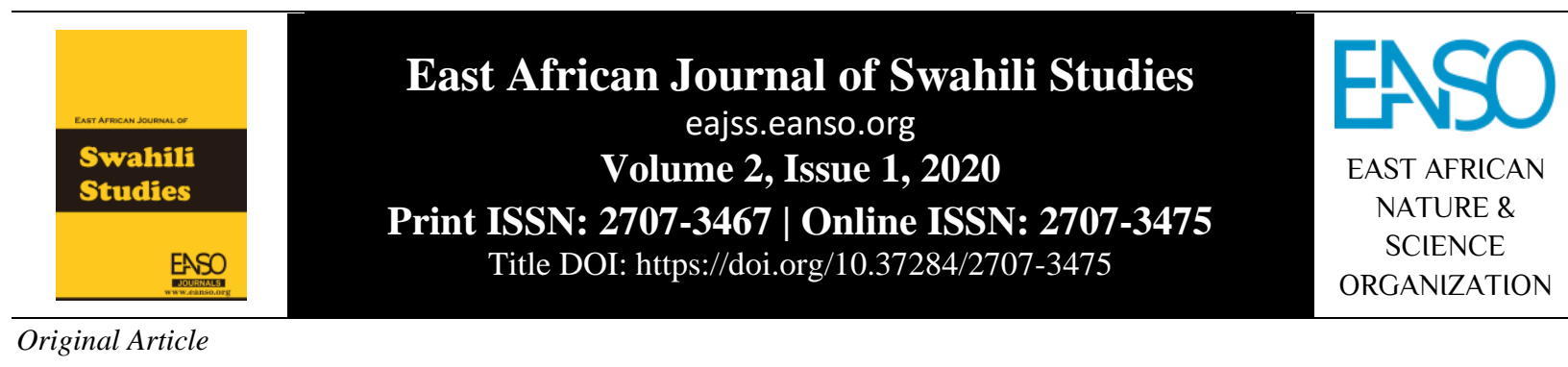

\title{
Vipengele vya Uwasilishaji wa Wahusika wa Kimazingaombwe na Uhusika: Mifano katika Watu wa Gehenna na Babu Alipofufuka
}

\author{
Anne C. Kenduiywa ${ }^{1 *}$, Prof. Issa Mwamzandi ${ }^{1} \&$ Dkt. Simiyu Kisurulia ${ }^{1}$ \\ ${ }^{1}$ Chuo Kikuu cha Kabianga, S.L.P 2030 - 20200, Kericho, Kenya. \\ * Author for Correspondence email: kenlyguide@gmail.com
}

Article DOI: https://doi.org/10.37284/eajss.2.1.49

\section{Article history:}

Received: 21 Oct 2019

Accepted: 30 Oct 2019

Published: 09 Apr 2020

Keywords:

Uwasilishaji,

Uhusika,

Umazingaombwe,

Babu Alipofufuka,

Watu wa Gehenna.

\section{IKISIRI}

Makala haya yanatathmini uwasilishaji wa wahusika wa kimazingaombwe na uhusika katika kazi za Kiswahili. Uchanganuzi huu ni wa kimaktaba tukizingatia mihimili ya nadharia ya Umazingaombwe. Riwaya za $B a b u$ Alipofufuka na Watu wa Gehenna zilichaguliwa kimakusudi kwa sababu uwasilishaji umechochewa na mbinu ajabuajabu za waandishi katika uhusika na usawirishaji wa wahusika kwa njia unaojenga dhamira ya kimazingaombwe. Uwasilishaji huu unazua utata kwa wasomaji katika kunata ujumbe unaowasilishwa na wahusika hao katika riwaya hizi teule za kimajaribio. Waandishi hawa, Wamitia na Olali, wanasukanisha uhalisia uliochongwa kwa njia maalum katika ruwaza inayobadilikabadilika daima, huku wakiwakilisha matukio ya kawaida na maelezo ya kina sambamba na vipengee vya kifantasia na kindoto na kwa kutumia mambo yaliyotokana na visasili na hekaya. Aidha, kazi hii ilidhamiria kuthibitisha uwasilishaji wa kiuhalisajabu katika mandari au mazingira ya kimazingaombwe. Utafiti huu umejaribu kujaliza mapengo yanayoleta matatizo ya ufahamu na usadikifu wa kazi tulizo teua za kihalisiajabu. Maandishi katika utafiti huu yanadhamiria kukuza na kupanua uelewekaji wa riwaya za kisasa mbali na kufaidi wasomaji mbalimbali na hata kuwapa motisha wahakiki kushughulikia zaidi wahusika, uhusika na uwasilishaji katika misingi ya umazingaombwe katika fasihi simulizi.

\section{APA CITATION}

Kenduiywa, A., Mwamzandi, I., \& Kisurulia, S. (2020). Vipengele vya Uwasilishaji wa Wahusika wa Kimazingaombwe na Uhusika: Mifano katika Watu wa Gehenna na Babu Alipofufuka. East African Journal of Swahili Studies, 2(1), 1-7. https://doi.org/10.37284/eajss.2.1.49. 


\section{CHICAGO CITATION}

Kenduiywa, Anne, Issa Mwamzandi, and Simiyu Kisurulia. 2020. "Vipengele Vya Uwasilishaji Wa Wahusika Wa Kimazingaombwe Na Uhusika: Mifano Katika Watu Wa Gehenna Na Babu Alipofufuka". East African Journal of Swahili Studies 2 (1), 1-7. https://doi.org/10.37284/eajss.2.1.49.

\section{HARVARD CITATION}

Kenduiywa, A., Mwamzandi, I. and Kisurulia, S. (2020) "Vipengele vya Uwasilishaji wa Wahusika wa Kimazingaombwe na Uhusika: Mifano katika Watu wa Gehenna na Babu Alipofufuka”, East African Journal of Swahili Studies, 2(1), pp. 1-7. doi: 10.37284/eajss.2.1.49.

\section{IEEE CITATION}

A. Kenduiywa, I. Mwamzandi, and S. Kisurulia, "Vipengele vya Uwasilishaji wa Wahusika wa Kimazingaombwe na Uhusika: Mifano katika Watu wa Gehenna na Babu Alipofufuka”, EAJSS, vol. 2, no. 1, pp. 1-7, Apr. 2020.

\section{MLA CITATION}

Kenduiywa, Anne, Issa Mwamzandi, and Simiyu Kisurulia. "Vipengele Vya Uwasilishaji Wa Wahusika Wa Kimazingaombwe Na Uhusika: Mifano Katika Watu Wa Gehenna Na Babu Alipofufuka". East African Journal of Swahili Studies, Vol. 2, no. 1, Apr. 2020, pp. 1-7, doi:10.37284/eajss.2.1.49.

\section{UTANGULIZI}

Uwasilishaji ni dhana inayotumiwa kuelezea njia au mtindo unaotumiwa na wasanii wa fasihi katika upazaji wa ujumbe hadi iwafikie walengwa wao (Wamitila, 2003). Umazingaombwe ni wazo linalotumiwa na waandishi wa fasihi katika uhusika na kwa kusawiri wahusika kwa njia ambayo inawawezesha kusababisha na kutekeleza matukio ya kiajabu. Akieleza kuhusu jambo hili, Mbathia (2002) anafafanua kuwa umazingaombwe ni nadharia mojawapo inayotumika kwa kiwango kikubwa katika kazi ya fasihi inayojengwa kutokana na matumizi ya mbinu changamano katika uhusika na usawirishaji wa wahusika.

Kwa mujibu wa Wamitila (2003), uwasilishi katika umazingaombwe ni dhana inayotumiwa katika kuelezea njia ya kuwasilishia maana katika kazi ya kifasihi lengo likiwa kufikisha mawazo la mwandishi hadi kwa wanaolengwa kwa kushughulisha wahusika wa aina mbalimbali wenye mawasiliano na matukio ya kiajabu. Kulingana na nadharia ya uhalisiamazingaombwe, fantasia au uhalisiajabu hutumia taswira ili kujengea msomaji picha mawazoni. Mfano ni katika riwaya ya Adili na Nduguze (uk 35), tunapoelezwa kuhusu watu wanaokuwa na uwezo wa kubadilishwa na kufanywa kuwa manyani jambo linalomfanya msomaji ajijengee picha akilini mwake na kutafakari umazingaombwe wa watu hao. Mambo katika fasihi aina hii huonekana yakiingiliana katika ukubalifu fulani kuambatana na ubunifu wa kimiujiza wa mwandishi akiwa na lengo la kupasha ujumbe wake kwa njia ya kutatanisha. Matukio ya wahusika wa kimazingaombwe ni ya kusawiriwa kiajabuajabu, ama kwa njia ya kuogofya na hata ya kutisha na kubabaisha msomaji. Matukio haya hayawezi kuashiria hali halisi ya maisha katika jamii. Mtindo unaotumika katika uhusika na katika uwasilishaji wa ujumbe wa kimazingaombwe unavunja sheria na kanuni ambazo zinatumika katika fasihi za kimapokeo au katika usawiri unaozingatia desturi ya kawaida katika jamii husika.

S. A. Mohamed ni msanii aliyeandika riwaya ya Babu Alipofufuka akizingatia matumishi ya uhalisiamazingaombwe katika usawirishaji wa wahusika wake. Hii ni riwaya iliyoandikwa kwa mtindo wa kifantasia katika hali mbalimbali ambapo kuna kinyume fulani katika usemi, hali au matukio. Dhana hii ya kifantasia husababisha hali inayokwenda kinyume na matarajio ya msomaji (Wamitila, 2003, uk 79). Umazingaombwe wa wahusika katika riwaya hii unatokana na matendo ya kiajabu yanayotekelezwa na wahusika wa kimazingaombwe wanaowakilisha watawala wabaya katika jamii. Maelezo katika hadithi nzima inatolewa na wahusika waliosawiriwa kwa njia ya kifantasia na wenye matukio na uwasilishaji wa kimazingaombwe katika mazingira yasiyo ya kawaida jambo ambalo huwaletea wasomaji utata katika kusoma na kunata ujumbe kwa ukamilifu.

Wamitila (2002, uk 18) akitoa maelezo yake, anasema kuwa wahusika ni viumbe ambao majukumu yao ni kutekeleza shughuli za mtunzi katika fasihi. Wahusika hao huchukua sehemu kubwa katika kazi nzima ya kifasihi na wana sifa 
zinazowatambulisha kulingana na maadili, itikadi na falsafa zao. Hivi ni kusema kuwa waandishi husawiri wahusika wao kuambatana na yale wanayoyasema katika mazungumzo na yale wanayoyatenda kulingana na matendo au matukio yao. Msingi wa uhusika na uwasilishaji katika riwaya hizi ndiyo kiini na motisha ya uhamasishaji wa wahusika hao. Wajibu kuu ya wahusika katika fasihi ni kuwasilisha ujumbe kwa kushirikisha matendo, hisia, mazungumzo na mawazo wa mwandishi.

Riwaya ya Watu wa Gehenna iliyoandikwa na Tom Olali, ni riwaya ya kimajaribio yenye uwasilishi tata na hadithi ya kugutua na kukanganya mawazo ya msomaji. Usawiri wa wahusika katika riwaya hii unatekelezwa kwa kutumia mtindo ambao unakiuka taratibu na uzoefu wa kawaida katika uandishi wa riwaya za kiuhalisia. Jambo ambalo huweza kumfanya msomaji wa riwaya hi kujipata katika hali ya kutoelewa, kufumbwa au kufungwafungwa bila kufaulu kutatanua au kutathmini atakavyo weka imani yake kulingana na ujumbe anaoupata anaposoma riwaya hii. Nia ya mwandishi ni kwamba msomaji anajihisi kukosolewa, kuchokozwa na kung'ang'ana kujiepusha na uwezekano wa kuwa mmoja ya watu wa Gehenna! Mbali na kufichua mambo mabaya yanayofanyika katika jamii kama vile uuzaji wa sehemu nyeti za zeruzeru, kutatizwa kwa wafanyakazi mbalimbali katika uongozi wa mabeberu, uhusika katika riwaya hii umetekelezwa kwa njia tatanishi chungu nzima.

Waandishi hawa, Said Ahmed Mohamed na Tom Olali wamesawiri wahusika wa kimazingaombwe wananuiwa kuwasilisha ujumbe katika riwaya kwa njia inayotia kitumbo maji. Riwaya hizi pia hujulikana kama riwaya mpya za kimajaribio ambazo zinabuniwa kuashiria uhalisiajabu unaopatika katika jamii. Kulingana Wamitila (2003, uk 180), riwaya za kimajaribio hutumika kwa kuelezea riwaya za kimazingaombwe ambayo inakiuka kanuni au desturi za kawaida katika uandishi wa riwaya za kawaida. Wamitila akiendelea kuelezea kuhusu riwaya hizi, anafafanua kwamba riwaya hizi mpya pia hushughulikiwa vilivyo na wasanii wa usasaleo wakiwa na lengo la kubuni wahusika wenye ujumbe unaobeba matendo ya kushtua na kutia hofu. Akiendelea kufafanua Wamitila nasema kuwa, waandishi wa aina hii huwa na nia ya kujitenga na mitindo za kawaida za uandishi wa riwaya (2003, uk 180).

\section{UWASILISHAJI WA WAHUSIKA}

Katika kushughulikia lengo mojawapo la utafiti huu, waandishi walichanganua sifa za wahusika wa kimazingaombwe pamoja na kuchunguza matendo yanayowafanya wahusika hao waonekane viumbe visivyo vya kawaida ili kuthibitisha umazingaombwe wao. Wahusika huwa nguzo kuu katika kuleta wazi mawazo ya mtunzi na kusababisha mawasiliano katika kazi ya kisanaa. Kupitia kwa mshikamano wa wahusika hao wanapotangusana na kuvunja kanuni au maafikiano mbalimbali na wahusika wengine Tumebainisha upekee na umuhimu wa kila mmoja wao katika kuendeleza ama kuonyesha sifa bainifu za kiuhalisiajabu kama zinavyojitokeza katika riwaya zetu.

Uhusika katika riwaya hizi zetu teule ulijengeka kutokana na hadithi za kawaida katika jamii zetu. Watunzi wanaosawiri wahusika kwa kutumia mbinu za kimazingaombwe wanafanya hivyo kwa kuiga mbinu za fasihi simulizi zilizokuwa zikitolewa na kusambazwa kutoka kizazi kimoja hadi kingine kwa kusimuliwa ama kwa kupitia mazungumzo katika jamii. Ni simulizi ambayo wahusika kama vile majitu, mizimu na hata wanyama waliaminika kuwa walikuwa na uwezo wa kutangamana na wanadamu katika ulimwengu halisi. Katika kipengele cha uwasilishaji wa wahusika hao wa kimazingaombwe, tumetazama matendo yao yanayojitokeza kulingana na mandari ambayo yanabuniwa vilivyo na watunzi wakujaribu kuambatisha matendo ya wahusika hao na mahali anapotekelezea matendo hayo. Kupitia uchunguzi huu, tumebainisha mahali panapojengwa na mnamotokea matukio hayo ya aina yoyote yawe yanafanywa na wahusika wa kawaida au wahusika wa kimazingaombwe.

Katika uchunguzi wetu kuhusu mandhari, tumefanikiwa kufahamu kuwa ndoto pia ni aina ya mojawapo wa mandhari ambayo ilichukua nafasi kubwa katika riwaya za Babu Alipofufuka na Watu wa Gehenna. Akieleza kuhusu ndoto, Malcolm (1959) anasema kuwa ndoto katika uwasilishaji wa ujumbe ni mtiririko wa mawazo yanayobebwa akilini mwa mtunzi. Matukio haya yote 
yanayotekelezwa na wahusika katika fasihi ni mambo katika mawazoni mwa watunzi ambayo yanasawiriwa yaambatane na maono, taswira na matukio yanayodhihirisha umazingaombwe wa wahusika. Hivyo basi ndoto ni chombo kinachotumiwa kusafirisha hisia na sifa za wahusika wanaosawiriwa na mtunzi na zinazojengeka katika mandhari husika.

Tukiangazia uwasilishaji wa wahusika wa kimazingaombwe, ilibidi tuangalie aina mbalimbali ya mandhari ambayo mwandishi Olali katika kitabu chake cha Watu wa Gehenna alibuni na kutumia katika kazi yake ya usanii. Tulichanganua aina mbalimbali ya mazingira ambamo matukio ya kimazingaombwe yalitekelezewa na wahusika wa kimazingaombwe.

Mandharia au mazingira ni istilahi inayotumika katika kuelezea muktadha au mahali ambapo hadithi au tukio la mhusika linatekelezewa katika kazi ya kisanaa. Dhana hii pia huweka mwanga hali ya aina mbalimbali ya mazingira yanayopatikana katika jamii husika. Katika kufafanua zaidi, waandishi katika sanaa wamesawiri wahusika wa kimazingaombwe kisha kubuni mandhari ya kimiujiza ambayo wahusika hao wa kifantasia huweza kutekeleza matukio ya kiajabu wakiwa katika mandhari ya kawaida na wakati mwingine inaweza kuwa matendo yao yanaonekana kama ya kawaida lakini mazingira ni ya kimiujiza na basi hali ya kimazingaombwe kuibuka. Mbinu zinazotumiwa katika uhusika na katika usawiri zinatumika kwa njia ya kiajabu kana kwamba matendo ya wahusika yanaambatishwa vilivyo na mandhari ambapo shughuli hizo za wahusika hufanyiwa (Wamitila, 2003, uk 108-114).

Mwandishi wa riwaya ya Watu wa Gehenna katika uhusika na usawiri alibuni mandhari ambayo mengine ni ya kimiujiza. Amesawiri wahusika wake watekeleze matukio ya kifantasia ambayo yanaingiliana vizuri kulingana na hali ya matendo yao. Katika kitabu chake cha Watu wa Gehenna, Olali alitumia mbinu kimakusudi ambazo zimesababisha uhusika na usawiri katika kazi yake inaoashiria uwasilishaji wa ujumbe unaozalisha hali ya kimazingaombwe. Wasomaji katika kazi hii huchorewa taswira ya kimazingaombwe na ya kutatanisha kupitia kwa matukio ya wahusika wa kimazingaombwe kama vile maisha katika jehanamu mahali watu wanahukumiwa. Mtunzi amesawiri na kupapea jina Gehenna mahali ambapo ni pa kuadhibu na kupatisha taabu au maumivu makali watu walioshiriki matendo maovu wakiwa hai katika jamii. Mwandishi amesawiri Gehenna kama eneo ambalo lina moto mkali usiokwisha wa kutesea wafanya dhambi milele na milele (uk 1-8).

Kwa kutumia mbinu za kimiujiza yasiyoeleweka katika uhusika, Olali alisawiri wahusika wake wa kimazingaombwe na kuwaweka katika mazingira tata na ya kiajabu yenye matukio ya kustaajabisha ambayo watu hasa viongozi walishiriki katika matendo mabaya katika jamii na mwishowe wanaadhibiwa kwa kugeuzwa watu ambao mazingira yao ni gereza lile milele. Olali, pia alisawiri na kutumia mandhari haya ya kimazingaombwe yenye moto unaowaka daima ukichoma na kutaabisha watu bila kikomo, mtunzi huyu anadamiria kuashiria hukumu kwa viongozi waovu katika jamii. Mtunzi pia anatumia hali hii kama njia ya kuleta onyo kwa viongozi hata wa kisasa katika jamii wanaotenda matendo mabaya yanayosababisha mahangaiko mabaya kwa wananchi wa kawaida. Mtunzi pia anawaonya na kuwahakikishia viongozi hao kuwa mwishowe hao watapata adhabu kali kwa kutowajibika vilivyo.

Kutokana na umazingaombwe katika uhusika na katika usawiri wa wahusika wanaowekwa kwenye mandhari ya kiajabuajabu, tunaona utumizi wa mbinu za nadharia ya umazingaombwe ikijitokeza. Wahusika wamesawiriwa kuvuka mipaka na hivyo mwandishi anapata uwezo ama uhuru wa kutoa maudhui ya ulimwengu nzima bila kulazimika kufuata sheria fulani ya kifasihi. Mwandishi huyu kutokana na uwasilishaji wa wahusika wa kimazingaombwe, amesawiri umazingaombwe huu akionyesha kwamba ni wazi kuwa mambo mengi ya kifantasia yanaweza kufanyika mawazoni mwa mtu lakini matokeo yake yanaweza kumdhuru bado kulingana na imani yake.

Mtunzi amesawiri pia wahusika wenye matukio ya kimazingaombwe yanayofanyika katika vyuo vikuu na hata katika shule za upili. Anatumia fursa hii kusababisha uwasilishaji unaohusu mambo yanayoweza kutokea katika jamii yetu ili kuwawezesha wahusika wake kuelimisha jamii hasa wanafunzi wasije wakajihusisha na mambo yatakayo haribu mielekeo yao ya kimasomo na hata 
kimaisha kwa jumla. Anawaambia kwamba kukiwa na jambo la lazima linalowalazimisha wafanye maandamano, basi wafanye hivyo kwa uagalivu mno. Mtunzi akifanya usawiri wa aina hii pia alilenga kuleta funzo kupitia wasomaji utakao enea kindanindani na mwishowe kuweka kikomo tabia zinazohusu mipango ya maandamano katika vio mbalimbali hasa za nchi za kiafrika. Jambo ambalo lingali likifanyika hata wakati wa leo (uk 121-123).

Tumepata kuona umuhimu ya uwasilishaji wa aina hii pia katika kuelimisha jamii nzima wakiwemo watu wazima hasa kuhusu migomo yanayofanyika mara kwa mara katika viwanda mbalimbali. Vitendo vya migomo wakati mwingine husababisha wafanyikazi hasa katika makampuni ya watu au vikundi fulani vya kibinafsi kupoteza kazi kabisa. Kwa mfano makampuni barani Afrika yanayohusishwa na wachuma chai na pia makapuni ya ulimaji wa kahawa na makampuni mengine mengi katika nchi tofauti za Kiafrika na mwishowe tunaona kuwa nchi nyingi zinasababishwa kuanguka au kusimamisha makapuni hayo moja baada ya nyingine bila mpangilio.

Mtunzi katika usawiri na uhusika alibandika katika mawazo wa msomaji taswira ya kimazingaombwe. Mwandishi anafanya hivo kwa kusawiri uwasilishaji utekelezwe kwa njia ya ajabu na wahusika wa kimazingaombwe na kuwawezesha wafanye matukio ya kimiujiza yanayosababisha waziwazi na hata kutia wayowayo kwa wasomaji kwani wa aina hii uwasilishaji una beba ujumbe wenye matukio yasiyowezekana wala kuaminika katika hali ya jamii ya kawaida. Ni matukio ya kimazingaombwe pia tunapopata kuona jinsi waandishi katika kazi za kifantasia wanavyobuni wahusika wa kimazingaombwe kisha kuwasawiri watekeleze matendo ya kimiujiza kupitia matumizi ya mbinu za kiajabu katika uhusika na katika usawiri wa wahusika.

La ajabu zaidi ni jinsi wanavyomsawiri mhusika mmoja kuwa na uwezo wa kubadilikabadilika na kuchukua nafsi ya mhusika mwingine kisha kutekeleza matukio ya kiajabu. Mtunzi Olali katika riwaya yake amesawiri wahusika wake wa kimazingaombwe na kuwapa jukumu la kulinda gereza na kusimamia mahitaji yote ya kiajabu yanayohusu wanadamu. Jambo la kimiujiza ni kuwa wahusika hao wamesawiriwa kuwa na uwezo wa kubadilikabadilika vilivyo kuligana na jinsi wanavyotakikana wawe ndio waweze kushughulikia jambo fulani. Wakiwa na shughuli ya kufanya huko angani mara moja wanamea mabawa na kupata uwezo wa kuruka na hata kuishi hewani kwa muda wote wanaotakikana wawe angani. Wakiwa na shughuli ya kufanya baharini wanajigeuza meli, nyangumi ama mnyama yeyote wa baharini. Ajabu tena ni kuwa wahusika hao wa kimazingaombwe wamesawiriwa wawe na uwezo wa kumulika kila mahali ulimwenguni wakitafuta watu wamchaye Mungu. Wanapowapata, wanawapangia kwa njia moja ama lingine wafe. Wanafanya hivyo kwa kuwapangia ajali za barabarani, za ndege au vifo vya kutokana na mizozo ya kikabila katika jamii. Wahusika hao wa mahakamani wamepewa vyeo mbalimbali na kila kikundi kina jukumu lake na wanasawiriwa kuwa na uwezo wa kutatua mahitaji yao yote kulingana na mpangilio wa jaji wao mkuu (uk 187-189).

Mtunzi alitumia umazingaombwe kwa kumshirikisha msomaji na kututuliza moyo anapouliza kama anafikiri ilikuwa ni ndoto au nini (uk 189). Mwandishi pia kwa kutumia uhalisiajabu katika ndoto ya aina hii alimulika uovu unaotekelezwa na viongozi mashuhuri kufanya maovu ya kimazingaombwe. Wanasiasa, wafanyi biashara na watu waovu wanatumia viwiliwili vya zeruzeru kupigia ramli ili wajitajirishe huku wakipuuza haki za zeruzeru na kuwaona hao si chochote si lolote na hata kufikiria kwamba hao sio binadamu kamili. Katika mahakama hii, mavazi mekundu kama damu yanayovaliwa na mahakimu na majonzi ya damu yanatiririka na washukiwa yanadhihirisha kuwa kuna mashaka na majuto kali.

\section{Vipengele Vinavyojenga Uwasilishaji wa Wahusika wa Kimazingaombwe}

Katika kufafanua zaidi umazingaombwe wa wahusika katika riwaya tulizoziteua za Watu wa Gehenna na Babu Alipofufuka tumechunguza yaliyoandikwa kuhusu mbinu walizozitumia watunzi wa riwaya hizi katika uhusika, usawiri, na jinsi wahusika hao wanawasilisha ujumbe wao kwa njia ya kimazingaombwe.

Kulingana na Wamitila (2003, uk 278), uhusika ni dhana au istilahi ambayo hutumiwa kuelezea njia na mikakati inayotumiwa na wasanii katika 
usawirishaji wa wahusika katika kazi zao. Baadhi ya wasanii hutilia uzito jambo hili la uhusika. Wamitila anaendelea kufafanua kuwa waandishi hawa huweka imani yao wakidai kuwa kilicho na uzito na maana kabisa katika usanii ni jinsi matendo au matukio yanayofanyika katika fasihi yanavyowaathiri wahusika. Kulingana na Wamitila, athari hiyo huwa na matokeo mengi tofautitofauti kuhusu mikabala yao yanayoenda sambamba na matukio yaliyojengeka kutokana na aina ya mbinu zilizotumiwa katika usawiri. Mfano ni katika matumizi majina au mbinu ya majazi katika kutekeleza kazi ya kimazingaombwe katika fasihi.

Njogu na Chimerah (1999:250) katika kuchangia ufafanuzi zaidi kuhusu dhana ya uhusika wanasema:

'Uhusika wa jadi umejengwa kwenye usawirishaji wa wahusika kwa msingi wa uhalisi wa kijamii. Wahusika wa jadi au wa kimapokeo wanakaribiana na binadamu wanaopatikana katika ulimwengu wa kawaida.'

Katika kuangazia uwasilishaji wa wahusika, Wamitila (2003, uk 123) anafafanua dhana ya wahusika kama ifuatavyo:

'Mhusika ni kiumbe mtenda kazi katika kazi ya kisanaa na ambaye ana uwezo wa kuiga mienendo ya binadamu kwa kiasi kikubwa. Wasifu wa mhusika unategemea mbinu za mtunzi katika usawiri na taratibu za uhusika zinazomfanya awe na uwezo wa kutekeleza matukio kulingana na mpangilio wa mawazo ya mtunzi katika jamii husika. Kunawezekana kuwa matendo ya wahusika yanayoelekezwa na mtunzi katika mitazamo ya kihalisia yanamsawiri wahusika kwa njia inayomfanya afanane sana na binadamu katika ulimwengu wa kawaida ingawa matukio yanayotokea yaweza kuwa ya kimazingaombwe.'

Akielezea dhana ya wahusika, Wamitila (2008, uk 308-369) anaeleza kwamba, wahusika wowote ya kifasihi ni nyenzo kuu. Hii ni kwa sababu wao ndio wanaoendesha kazi zote na kuzalisha matukio yanayopatikana katika kazi ya kifasihi inayohusika.
Mtazamo wa dhana ya wahusika unaweza kutofautiana kutegemea mtindo na utaratibu unaofuatwa au anaouchukua mhakiki au mwandishi na pia kulingana na nadharia ya fasihi inayohusishwa. Mhusika katika fasihi ni kiumbe mtendaji katika kazi ya kisanaa na huwa kielelezo cha kiumbe au viumbe wanaopatikana ulimwenguni na shughuli ya kila mmoja wao yakifanywa kulingana na matarajio yaliyo nayo watunzi mawazoni mwao wawe wahusika wa kimazingaombwe au wa kihalisia.

Tukifafanua zaidi, mhusika wa kihalisia ana sifa nyingi zinazohusishwa na binadamu katika maisha ya kawaida au ya kila siku. Kisha kuna mhusika wa kimazingaombwe ambaye anahusishwa kwa kiasi kikubwa na njozi au fantasia. Mhusika wa aina hii hupatikana katika mazingira ajabuajabu au wenye sifa za ulimbwende anaposhikamana na viumbe wasiokuwa wa kawaida. Njozi hutumiwa kueleza kazi ya fasihi ambayo huonyesha anaowaza msanii kwa kiwango cha juu cha matukio ya kimazingaombwe. Dhana ya njozi ni pana sana na huweza kuambatisha bunilizi za kisayansi na kazi za kiuhalisiajabu ambazo zinahusishwa na matukio ya kimazingaombwe.

Kuhusu usawiri wa wahusika wa kimazingaombwe, Wamitila (2003, uk 312-313) anasema kuwa umazingaombwe hutumiwa kuelezea jinsi wahusika wa kifasihi walivyochorwa au wanavyowasilishwa na msanii au mtunzi wa fasihi katika kazi fulani. Usawiri wa wahusika hufanywa kwa kuhusisha majina yao, hisia zao, lugha wanazopewa na matumizi yao ya maneno na kwa kuwatambulisha kwa matendo yao wenyewe. Mbali na kukagua upimishaji wa nadharia chukulizi katika utafiti wetu, mwega au mwimo wa uchunguzi wetu umeweza kuthibitisha uwasilishaji wa ujumbe wa wahusika wa kimazingaombwe unaochukua nafasi kubwa katika uhusika na pia katika usawirishaji katika kazi zetu tukizingatia riwaya za Babu Alipofufuka na Watu wa Gehenna. Umazingaombwe wa wahusika wao wa kifantasia umefanyika kuambatana na malengo ya waandishi wa kazi hizi za Ahamed na Tom. Wahusika wametekeleza uwasilishaji wao vilivyo kuambatana na mazingira ya matukio hayo ya kimazingaombwe. 
Mandhari au mazingira ya kijozi katika riwaya zetu teule yamesawiriwa kwa kutumia mbinu za kipekee katika uhusika na kwa kufunganishwa vilivyo na mihimili ya kimazingaombwe katika uwasilishaji wa ujumbe walio nao watunzi mawazoni mwao. Ya kustaajabisha ni kwamba katika uchunguzi wetu tumeweza kufahamu kuwa ingawa uhusika na usawiri wa wahusika katika riwaya hizi zilifanyika nyakati mbalimbali tofauti, mbinu za uandishi zinaashiria nia ulio sawa kulingana na mawazo walio nao watunzi hao na kwamba tofauti zao za wanda wa utunzi sio kisingizio cha uelewano wa wasanii wa kazi hizi ambazo lengo lao ni kuafiki mahitaji ya kisanaa ya kisasa ya kimazingaombwe katika jamii. Hili ni jambo ambalo linaleta wazi kuwa utengano uliokuwa ukisababishwa na hali ya kifasihi umewekwa kando na hamna tena kwa sababu wasanii wa kisasa wako huru kushughulikia kazi zao za uhusika na za usawiri wa wahusika wa kimazingaombwe bila kufungwa na vikwazo vyovyote vyaa taratibu za kifasihi.

Watunzi katika kazi za kimazingaombwe hutumia mbinu katika uhusika ambazo ni za ubinafsi fulani kutegemea ubunifu wa kila msanii wa kimazingaombwe. Lengo moja walio nao wanasanii hao wa kimazingaombwe ni kusababisha uwasilishaji wa ujumbe wao unaoweza kushughulisha matakwa ya kila mtu katika jamii kwa kupitia matumizi ya mbinu za usawiri ambazo hazina mpangilio unaowakitisha kwenye sheria za sanaa za kimapokeo. Wasanii hao wamefaulu katika kutatua mambo ya kifantasia ambayo hayangeweza kutatuliwa kwa kuzingatia mipangilio katika sanaa yasio huru. Wasanii hao ambao wameegemea mbinu za kifantasia katika uwasilishaji walikabiliana na hali geni katika jamii jambo ambalo ni sababu mojawapo ya waandishi S. A. Mohamed na Olali Tom kubandukia mawazo yao geni kwenye uandishi wa mazingaombwe katika kazi zao.

\section{HITIMISHO}

Katika kutamatisha tumebaini kwamba dhana ya uhalisiamazingaombwe katika uhusika ndiyo mhimili mkuu wa dhamira katika riwaya za Babu Alipofufuka na Watu wa Gehenna. Kupitia mihimili hii ya Nadharia ya Umazingaombwe, matokeo ya utafiti wetu yameoyesha kuwa, uwasilishaji wa wahusika katika riwaya hizi ni wa kiuhalisiamazingaombwe kumaanisha kuwa nadharia tuliyotumai katika utafiti wetu imekuwa wa manufaa katika kufanikisha utekelezaji wa mada ya utafiti wetu. Kutokana na maelezo haya ya uwasilishaji wa kimazingaombwe, tumepata ya kuwa mwanadamu ako na upungufu wa uwezo wa kuyadhibiti hali na mahitaji ya mambo mengi yanayohusu maisha yake kibinafsi. Hivyo tumebainisha ya kuwa mwanadamu daima anashughulikia uwezekano wa kujipatia riziki kila uchao. Pia, tumepata ya kuwa katika uwasilishaji wa kimazingaombwe, watu waliokufa bado wangalipo na sisi katika hali tofauti na kwamba wanaendelea kuathiri mienendo ya watu walio hai kulingana na jinsi imani yao inavyowaelekeza.

\section{MAREJELEO}

Malcolm, N. (1959). Dreaming U.S.A. London: Routledge and Kegan Paul Ltd.

Mbatiah, M. (2001). Kamusi ya Fasihi. Nairobi: Standard Textbooks Graphics \& Publishers.

Olali, T. (2010). Structure and Anti-structure of a Swahili New Novel: A Stylistic Analysis of Babu Alipofufuka. The Nairobi Journal of Literature, UoN 82-98.

Wamitila, K.W. (2002). Bina-Adamu! Nairobi: Phoenix Publishers. 